\title{
Observation of microstructure change during freeze-drying by in- situ X-ray Computed Tomography
}

Nakagawa, K. ${ }^{a^{*}}$; Tamiya, S. ${ }^{\text {a }}$ Sakamoto, S. ${ }^{\text {a }}$ Do, G. ${ }^{\text {b }}$ Kono, S..$^{\text {; Ochiai, T. }}{ }^{\text {d }}$

${ }^{a}$ Division of Food Science and Biotechnology, Graduate School of Agriculture, Kyoto University, Sakyo-ku Kitashirakawa Oiwakecho, Kyoto 606-8502, Japan

${ }^{\mathrm{b}}$ College of Bioresource Science, Nihon University, Japan

c Research and Development Center, Mayekawa MFG. Co., Ltd., Japan

${ }^{\mathrm{d}}$ Research and Development Headquarters, Asahi Group Foods Ltd., Japan

*E-mail of the corresponding author: kyuya@kais.kyoto-u.ac.jp

\begin{abstract}
$X$-ray computed tomography technique was used to observe microstructure formation during freeze-drying. A specially designed vacuum freeze-drying stage was equipped at the X-ray CT stage, and the frozen and dried microstructures of dextrin solutions were successfully observed. It was confirmed that the many parts of the pore microstructures formed as a replica of the original ice microstructures, whereas some parts formed as a consequence of the dehydration dependent on the relaxation level of the glassy phases, suggesting that the post-freezing annealing is advantageous for avoiding quality loss that relates to the structural deformation of glassy matters.
\end{abstract}

Keywords: freeze-drying; X-ray CT; ice microstructure; glassy state 


\section{Introduction}

Freeze-drying is known as one of the best drying methods in terms of preservation of product qualities. Due to its higher processing cost than the other drying method, quality assurance is a critical issue to meet requirements of industries. Detailed knowledge on the phenomena occurring in a freeze-drying system plays an important role in further improvement of process design. Freeze-drying process consists of freezing and drying steps. When an aqueous solution is subjected to freezing, an ice crystal phase coincidently forms with the freezeconcentrated phase. In most cases of freeze-dried formulations, the freeze-concentrated phase transforms into a glassy phase, and the water content in the glassy phase can be related to the temperature. The glass transition temperature $\left(T_{\mathrm{g}}\right)$ of the maximally freeze-concentrated glassy phase is commonly denoted as $T_{\mathrm{g}}$, and this temperature has a critical importance in terms of carrying out an appropriate freeze-drying run. During drying step, water is mainly removed from the ice crystal phase by sublimation (i.e. primary drying). The removal of water coincidently forms dried layer, and the subsequently sublimated water vapor must transfer through the dried cake layer with porous microstructures. The mass transfer resistance in the cake layer is thus controlled by modifying the ice crystal morphologies. ${ }^{[1-3]}$ Water is also removed from the freeze-concentrated glassy phase by vaporization (i.e. secondary drying) at above $T^{\text {g }}$. The viscous flow of the glassy phase may cause the loss of the dried layer structure that is called as collapse. The occurrence of collapse is thus provoked by the excessive increase of the product temperature and/or decrease of the evaporation rate. ${ }^{[4-8]}$ Furthermore, it must be noted that the glassy phase in a rapidly frozen solution is not perfectly freeze-concentrated because of the formation of the non-crystalized water (i.e. vitrified water). ${ }^{[9-11]}$ Annealing over $T_{\mathrm{g}}$ reduces the fraction of the amorphous phase toward the completion of the freeze-concentration; this is called as relaxation (glassy phase relaxation). The degree of the relaxation relates to the $T_{\mathrm{g}}$ and viscosity of the glassy state, and it would affect the dehydration rate from the glassy phase and consequently the strength of the dried layer. Therefore, the freeze-dried microstructure forms not only in the freezing step but also in the drying step. The formation of freeze-dried microstructure in the sufficiently freeze-concentrated frozen solution would be different from the one in the immature frozen solution. Our interest in this study was thus to investigate the influence of the glassy state relaxation on the microstructure formation during freeze-drying.

X-ray computed tomography (CT) is a powerful tool to visualize microstructure of a material without sample destruction. In this study, X-ray CT was used to observe microstructure formation during freeze-drying. Our interests in this study is to investigate the primary and secondary drying behavior by using in-situ CT technique with monochromatized $\mathrm{X}$-ray from the synchrotron radiation. A freeze-drying system was set-up at the X-ray CT 
stage, and the microstructure change during vacuum freeze-drying of dextrin solution was observed.

\section{Materials and Methods}

\subsection{Materials}

Millipore-purified water was used for the sample preparation. Sucrose was purchased from Wako Pure Chemical Industries (Osaka, Japan). Dextrin was donated by Matsutani Chemical industry Co., Ltd. (Hyogo, Japan). Dextrin of which dextrose equivalent (DE) equal to 11 was used in this study. Glass transition temperatures of the maximally freeze-concentrated aqueous dextrin solution $\left(T_{\mathrm{g}}^{\mathrm{g}}\right)$ measured by DSC was around $-11^{\circ} \mathrm{C}$.

\subsection{Frozen sample preparation}

Aqueous dextrin solution with a concentration of $20 \%(\mathrm{w} / \mathrm{w})$ was prepared and filled in a sample stage. Sample stage was composed of a metal base (made by stainless steel) and a plastic tube (diameter $6 \mathrm{~mm}$, height $10 \mathrm{~mm}$ ). The tube was attached on the metal base to make a space for putting a solution. The bottom of a solution contacts to the metal part. The sample stage with a sample solution was placed on a cupper block pre-cooled in liquid nitrogen so as to freeze inner solution rapidly with keeping its direction of freezing from the bottom to the top. Frozen solutions were subsequently annealed at $-5^{\circ} \mathrm{C}$ in an electric cooling devise for selected duration (0-12 h). Frozen samples were then stored in a refrigerator at $-90^{\circ} \mathrm{C}$ until measurement.

\subsection{Synchrotron $X$-ray computed micro tomography}

X-ray computed tomography was carried out at synchrotron facility SPring-8 (BL19B2, Hyogo, Japan). Monochromatized X-ray was used for this measurement in order to obtain Xray attenuation linear coefficient from the tomograms. The X-ray energy was adjusted to 12.4 KeV by a double Si-crystal monochromator (net plane: (111)). As schematized in Fig. 1, the measurement stage was equipped with a turntable with a cold finger of which temperature was controllable by an external air blowing device in the range of -80 to $-10^{\circ} \mathrm{C}$. The sample stage with a frozen sample was rapidly fixed on the sample stage pre-cooled at around $-15^{\circ} \mathrm{C}$. The sample stage on the turntable was then fully covered with a housing case, and the inner space was evacuated by connecting a vacuum pump in order to start freeze-drying. The top of this housing component was made by acrylic resin with a thickness of $0.5 \mathrm{~mm}$. The X-ray passed through this acrylic window and the sample, and then exposed to X-ray camera that was set $100 \mathrm{~mm}$ behind the housing component. An X-ray imaging unit (AA40, Hamamatsu Photonics K.K., Japan) and CCD camera (C4880-41S, Hamamatsu Photonics K.K., Japan) 
were used for image acquisition. 256 transmission images were acquired for one set of measurement by rotating the turntable from 0 deg to $180 \mathrm{deg}$ at a speed of $1.2 \mathrm{deg} / \mathrm{s}$. The exposure time of X-ray was set to $0.12 \mathrm{~s}$ for taking an image, and the pixel size in the image was $2.92 \mu \mathrm{m}$ square. A horizontal cross-sectional image (i.e. tomogram) was reconstructed from the transmission image by the filtered back projection method. Freeze-drying run was carried out with the sample stage temperature at around $-14^{\circ} \mathrm{C}$, inner pressure around $12 \mathrm{~Pa}$.

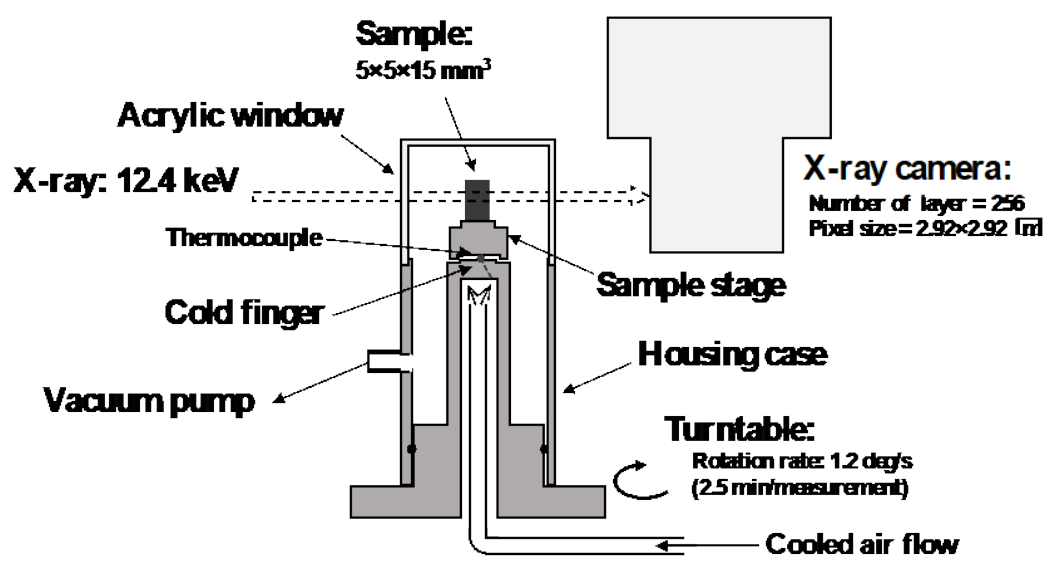

Fig. $1 X$-ray CT measurement set-up equipped with freeze-drying stage.

\section{Results and Discussions}

\subsection{CT images during freeze-drying}

The freeze-drying runs were carried out and CT images were successfully captured by the present system. First of all, CT images taken from the frozen zones were compared in Fig. 2. The boundary between the ice and freeze-concentrated phases was obviously formed in the sample prepared via longer annealing. One reason is due to the ice crystal size increase by Ostwald ripening mechanism. Another reason is the progress of the freeze-concentration due to the glassy state relaxation. The details of these kinetics during annealing were investigated in a former study of the authors [12], suggesting that both the glassy phase relaxation and Ostwald ripening jointly control the ice crystal growth/ripening kinetics, and the dominant mechanism was depending on the stage of annealing. Furthermore, the progress of freezeconcentration increases the density of the phase, this contribute to increase the attenuation level in the CT image and make clear contrast with the ice phase. It was also suggested that the dextrin-water system required more than 20 hours of annealing time for the completion of the glassy phase relaxation. 
Nakagawa, K.; Tamiya, S.; Sakamoto, S.; Do, G-S.; Kono, S.; Ochiai, T.
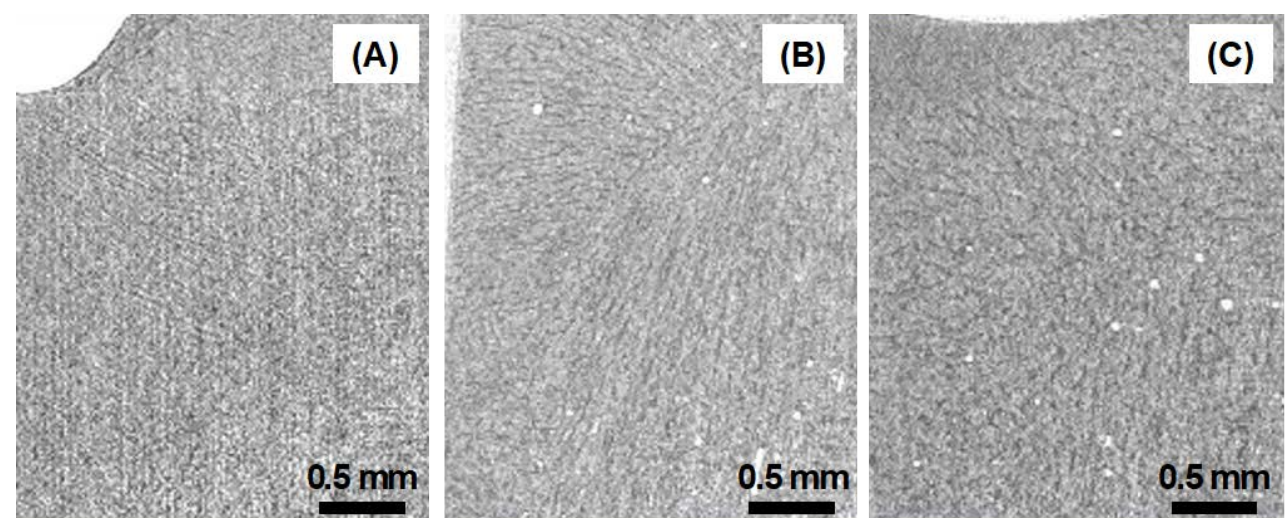

Fig. 2 X-ray CT images of frozen specimens (vertical cross sections); (A) Annealed for 0h, (B) Annealed for $12 \mathrm{~h}$, (C) Annealed for $30 \mathrm{~h}$.
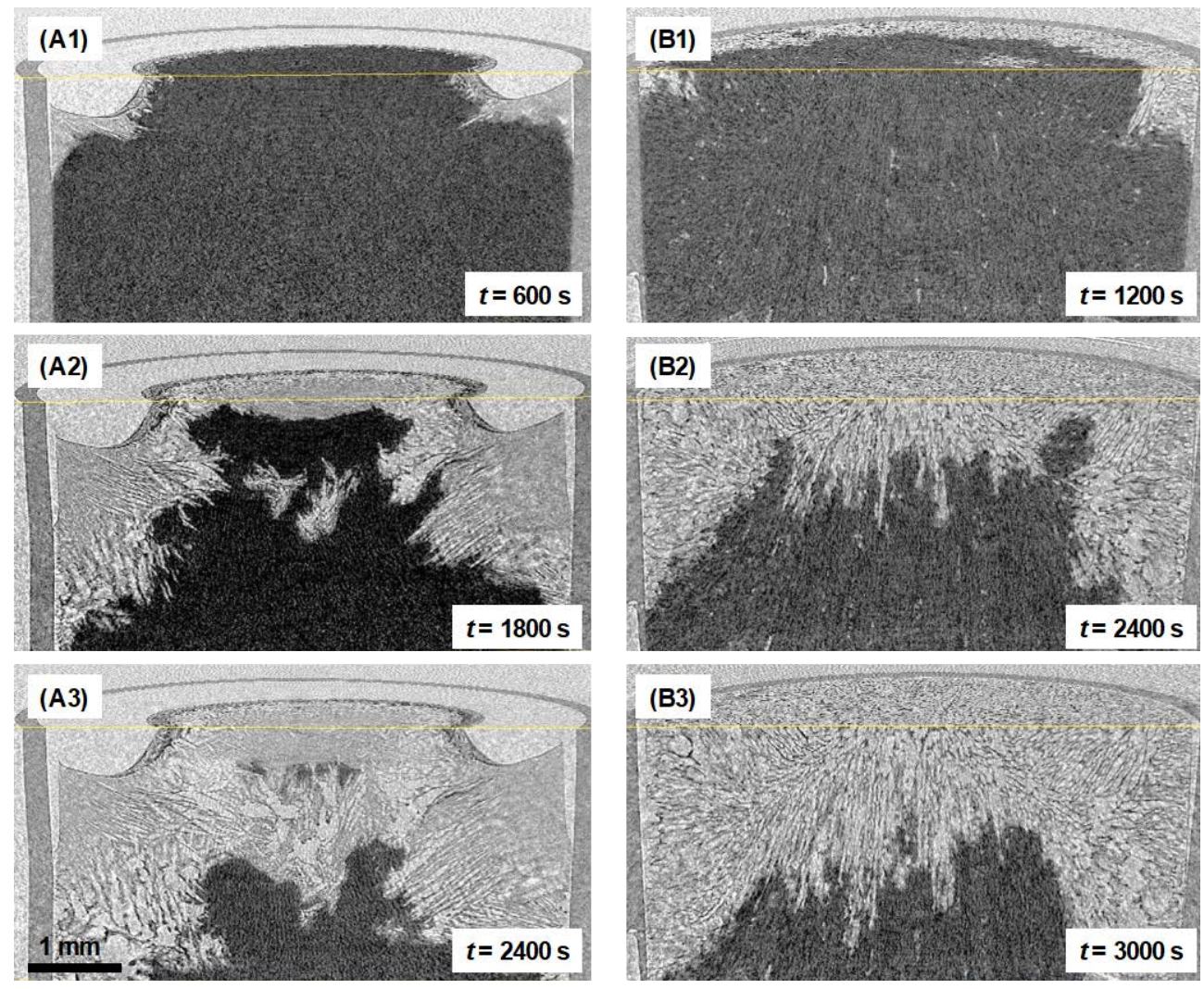

Fig. $3 X$-ray CT images of freeze-drying specimens (the duration under vacuum was stamped in the images); (A1-3) Annealed for $0 \mathrm{~h},(B 1-3)$ Annealed for $12 \mathrm{~h}$. 
3D reconstructed images of the samples during freeze-drying were shown in Fig. 3. It was visibly confirmed from the images of the sample via $12 \mathrm{~h}$ of annealing that the ice crystals were removed from the system by maintaining the original architectures made by the freezeconcentrated phase (Fig. 3A). This observation agrees with conventional explanation of a freeze-drying mechanism where a microstructure in a freeze-dried matrix is a replica of the frozen microstructure. On the other hand, this was hardly observed in the images of the sample prepared without annealing (Fig. 3B). Although extremely fine porous structures were formed at the top part, the formations of specific large pores were obvious in this drying system. These large pores were not visible in the frozen zone, and randomly distributed in the sample geometry. Considering that the glassy phase in the non-annealed solution was not perfectly freeze-concentrated, the apparent vapor pressure of this phase is higher than the perfectly freeze-concentrated phase. The apparent water vapor pressure, this is not equilibrium thermodynamic pressure, of the glassy phase is observed lower as the value of ( $T-T_{\mathrm{g}}$ ) become smaller. This is because the properties of glassy phase is kinetically controlled. ${ }^{[7]}$ Therefore, in the drying of non-annealed solution, water removal from this phase would be accelerated and easily lost its original microstructure.

\subsection{Fractal dimension and lacunarity}

The previous section discussed about the microstructure formation that occurred simultaneously with dehydration. In order to evaluate the differences in microstructures, the fractal dimension and the lacunarity of the porous microstructures were calculated from the images. Fractal dimension is a measure of structural roughness, whereas lacunarity is a measure of inhomogeneity or texture. In other words, lacunarity is the ratio of void space filled in a fractal structure. Cross sectional images were selected from the dried zone, and converted into binary images (Fig. 3). Image analysis was carried out on the binary images by a software FracLac version 2015 for ImageJ. The fractal dimension values for the nonannealed and annealed samples were both calculated as 1.8 . The lacunarity values, on the other hand, for the non-annealed and annealed samples were 0.14 and 0.12 , respectively. These values suggested that the structural difference between the non-annealed and annealed freeze-dried matrix was mainly due to the formation of the void space. The difference in the lacunarity values implies that the obvious void spaces seen in the non-annealed sample were not casted by ice crystals. Annealing in freeze-drying process has been recognized as a technique to reduce primary drying time and also to alter crystallinity and/or aggregation degree of the components ${ }^{[9,10,13-15]}$ The result of this study suggested that these advantages of annealing are realized by avoiding the structural deformation as seen in the freeze-drying of the non-annealed sample, and largely relate to the degree of the glassy phase relaxation. 

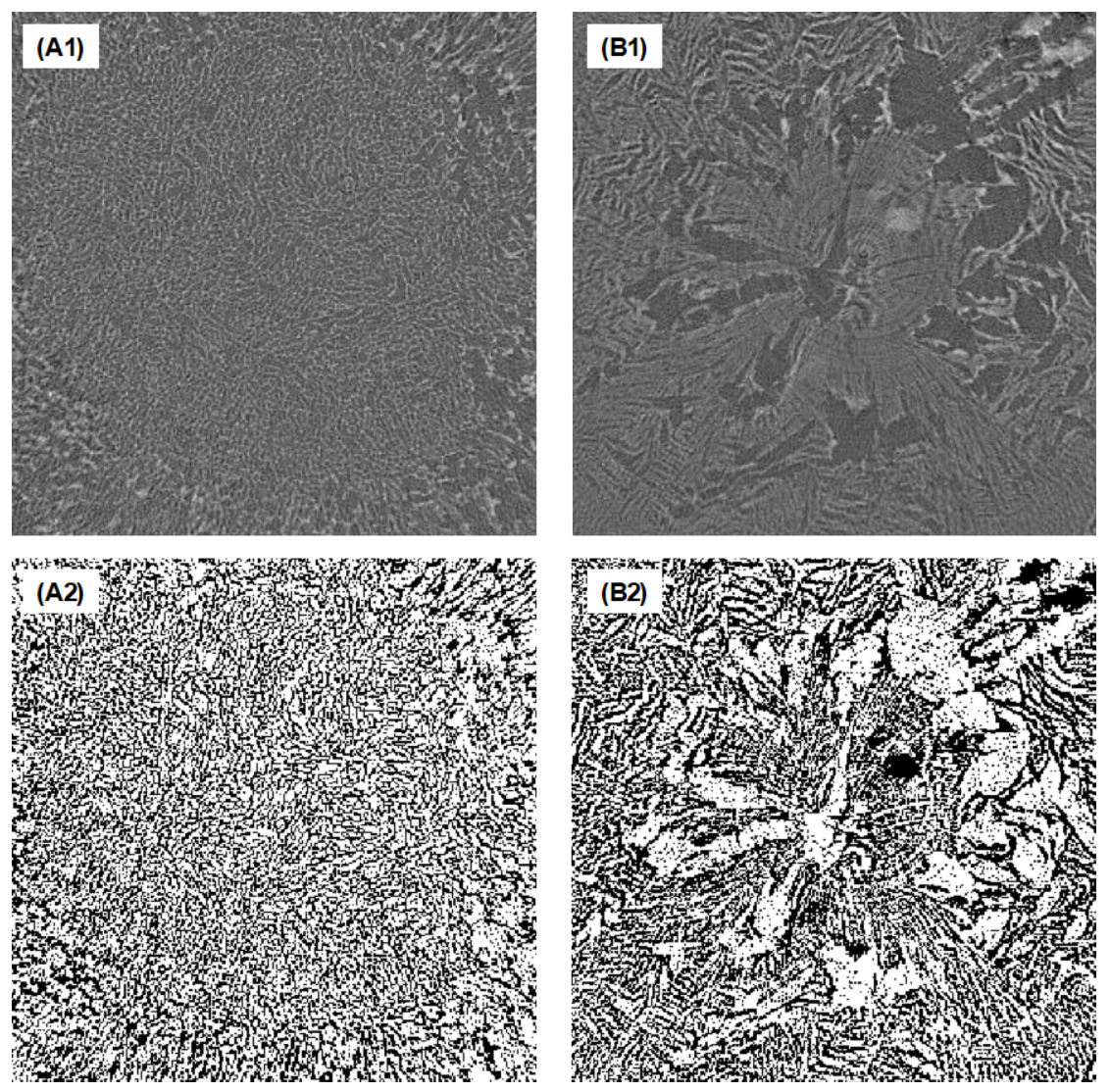

Fig. 4 X-ray CT images of freeze-dried specimens (horizontal cross sections); (A) Annealed for 0h, (B) Annealed for $12 \mathrm{~h}$. Binary images converted from A1 and B1 are shown at the bottom.

\section{Conclusions}

In-situ computed tomography technique with X-ray from the synchrotron radiation was used to observe microstructure formation during freeze-drying to investigate the primary and secondary drying behavior. A specially designed vacuum freeze-drying stage was equipped at the X-ray CT stage, and the frozen and dried microstructures of dextrin solutions were successfully observed. It was confirmed that the many parts of the pore microstructures formed as a replica of the original ice microstructures, whereas some parts formed as a consequence of the dehydration. This would relate to the relaxation level of the glassy phases. When rapidly frozen and non-annealed solution was subjected to freeze-drying, obvious deformation of the original ice microstructures could be provoked by the water removal from both ice and freeze-concentrated phases. Considering that the glassy phase in the nonannealed solution was not perfectly freeze-concentrated, water was rapidly removed from this phase and lost its original microstructure. It was suggested that the advantages of 
annealing are not only to reduce primary drying time due to the modification of ice crystal morphologies but also to avoid quality loss that relate to the structural deformation of the glassy matters.

\section{References}

[1] Searles, J.A.; Carpenter, J.F.; Randolph, T.W. Annealing to optimize the primary drying rate, reduce freezinginduced drying rate heterogeneity, and determine $\mathrm{Tg}^{\prime}$ in pharmaceutical lyophilization. Journal of Pharmaceutical Sciences 2001, 90, 872-887.

[2] Nakagawa, K.; Hottot, A.; Vessot, S.; Andrieu, J. Influence of controlled nucleation by ultrasounds on ice morphology of frozen formulations for pharmaceutical proteins freeze-drying. Chemical Engineering and Processing: Process Intensification 2006, 45, 783-791.

[3] Nakagawa, K.; Hottot, A.; Vessot, S.; Andrieu, J. Modeling of freezing step during freeze-drying of drugs in vials. AIChE Journal 2007, 53, 1362-1372.

[4] Slade, L.; Levine, H.; Ievolella, J.; Wang, M. The glassy state phenomenon in applications for the food industry: Application of the food polymer science approach to structure-function relationships of sucrose in cookie and cracker systems. Journal of the Science of Food and Agriculture 1993, 63, 133-176.

[5] To, E.C.; Flink, J.M. 'Collapse', a structural transition in freeze dried carbohydrates. International Journal of Food Science \& Technology 1978, 13, 583-594.

[6] Pikal, M.J.; Shah, S. The collapse temperature in freeze drying: Dependence on measurement methodology and rate of water removal from the glassy phase. International Journal of Pharmaceutics 1990, 62, 165-186.

[7] Slade, L.; Levine, H.; Reid, D.S. Beyond water activity: Recent advances based on an alternative approach to the assessment of food quality and safety. Critical Reviews in Food Science and Nutrition 1991, 30, 115-360.

[8] Levi, G.; Karel, M. Volumetric shrinkage (collapse) in freeze-dried carbohydrates above their glass transition temperature. Food Research International 1995, 28, 145-151.

[9] Sahagian, M.E.; Goff, H.D. Effect of freezing rate on the thermal, mechanical and physical aging properties of the glassy state in frozen sucrose solutions. Thermochimica Acta 1994, 246, 271-283.

[10] Ablett, S.; Izzard, M.J.; Lillford, P.J. Differential scanning calorimetric study of frozen sucrose and glycerol solutions. Journal of the Chemical Society, Faraday Transactions 1992, 88, 789-794.

[11]Levine, H.; Slade, L. Principles of “cryostabilization” technology from structure/property relationships of carbohydrate/water systems-a review. Cryo-letters 1988, 9, 21-63.

[12] Nakagawa, K.; Tamiya, S.; Do, G.; Kono, S.; Ochiai, T. Observation of glassy state relaxation during annealing of frozen sugar solutions by X-ray computed tomography. Eur J Pharm Biopharm 2018, 127, $279-287$.

[13]Kasper, J.C.; Friess, W. The freezing step in lyophilization: physico-chemical fundamentals, freezing methods and consequences on process performance and quality attributes of biopharmaceuticals. European Journal of Pharmaceutics and Biopharmaceutics 2011, 78, 248-263.

[14] Milton, N.; Pikal, M.J.; Roy, M.L.; Nail, S.L. Evaluation of Manometric Temperature Measurement as a Method of Monitoring Product Temperature During Lyophilization. PDA Journal of Pharmaceutical Science and Technology 1997, 51, 7-16.

[15] Goshima, H.; Do, G.; Nakagawa, K. Impact of Ice Morphology on Design Space of Pharmaceutical FreezeDrying. Journal of Pharmaceutical Sciences 2016, 105, 1920-1933. 